\title{
BI-RADS 3: Current and Future Use of Probably Benign
}

\author{
Karen A. Lee ${ }^{1,2} \cdot$ Nishi Talati $^{1,2} \cdot$ Rebecca Oudsema $^{1,2} \cdot$ Sharon Steinberger $^{1,2}$. \\ Laurie R. Margolies ${ }^{1,2}$
}

Published online: 27 January 2018

(C) The Author(s) 2018. This article is an open access publication

\begin{abstract}
Purpose of Review Probably benign (BI-RADS 3) causes confusion for interpreting physicians and referring physicians and can induce significant patient anxiety. The best uses and evidence for using this assessment category in mammography, breast ultrasound, and breast MRI will be reviewed; the reader will have a better understanding of how and when to use BI-RADS 3.

Recent Findings Interobserver variability in the use of BIRADS 3 has been documented. The 5th edition of the BIRADS atlas details the appropriate use of BI-RADS 3 for diagnostic mammography, ultrasound, and MRI, and discourages its use in screening mammography. Data mining, elastography, and diffusion weighted MRI have been evaluated to maximize the accuracy of BI-RADS 3.

Summary BI-RADS 3 is an evolving assessment category. When used properly, it reduces the number of benign
\end{abstract}

This article is part of the Topical collection on Breast Imaging.

Laurie R. Margolies

Laurie.Margolies@mountsinai.org

Karen A. Lee

Karen.Lee2@mountsinai.org

Nishi Talati

Nishi.Talati@mountsinai.org

Rebecca Oudsema

Rebecca.Oudsema@mountsinai.org

Sharon Steinberger

Sharon.Steinberger@mountsinai.org

1 Department of Radiology, The Mount Sinai Medical Center, 1176 Fifth Avenue, Box 1234, New York, NY 10029, USA

2 Department of Radiology, The Icahn School of Medicine at Mount Sinai, New York, NY, USA biopsies while allowing the breast imager to maintain a high sensitivity for the detection of early stage breast cancer.

Keywords BI-RADS 3 - Probably benign · Breast imaging reporting and data system · MRI · Mammography · Breast ultrasound $\cdot$ Breast cancer screening

\section{Introduction}

Efforts to improve the specificity and cost-effectiveness of screening mammography led to the development and widespread acceptance of short-term follow-up of probably benign findings. The purpose of the short-term follow-up algorithm is to reduce false-positive findings while retaining a high sensitivity for early stage breast cancer [1]. Probably benign (BI-RADS 3) has been formally established as a unique assessment category in the BI-RADS Atlas [2]. Designating a finding as probably benign in mammography is meant to indicate that the finding has a $2 \%$ or less chance of malignancy [3]. In practice, $0.9-7.9 \%$ of probably benign mammographic findings are upgraded to suspicious and proceed to biopsy [1, 4-6].

BI-RADS 3 is perhaps the most difficult of the assessment categories for the breast imager to properly use. Indeed, Michaels et al. have shown that there is considerable interobserver variability in the assessments of mammographic Bi-RADS 3 findings [7] and Grimm et al. have shown the same for MRI [8]. Ortiz-Perez has shown that formal instruction in the ultrasound BI-RADS lexicon improves the characterization of findings and BI-RADS assessments [9]. BI-RADS 1 and 2-normal and benignas well as BI-RADS 4 and 5-suspicious or highly suspicious-are relatively straightforward. BI-RADS 3 lurks, 
however, in the middle and has significantly different meanings for mammography, ultrasound, and MRI and indeed is audited differently for the three modalities. BIRADS 3 creates a wide variety of actions and reactions. It causes patient anxiety, eliminates some unneeded biopsies, and is often ignored by patients and referring clinicians. Radiologists who are not sure what to do with a finding often overuse BI-RADS 3. This paper will discuss the appropriate use of BI-RADS 3 with 3 core principles: (1) if a lesion is indeterminate or has worrisome features it is not BI-RADS 3; (2) BI-RADS 3 should not be used to delay diagnosis of a malignant appearing finding; (3) BI-RADS 3 should only be used after a full diagnostic workup.

The typical follow-up protocol for all modalities is similar. For mammography, for example, this includes the assignment of BI-RADS 3 at diagnostic imaging. At 6 months from the screening exam that prompted the recall, another diagnostic evaluation is completed and the finding is biopsied if warranted. Assuming the finding is stable, it is again assigned BI-RADS 3 and a bilateral mammogram in 6 months is performed. At 12 months from the screening exam, the diagnostic mammogram is repeated and is generally again assessed as BI-RADS 3 unless upgraded to BI-RADS 4 or 5. At the 12-month mark, although the exam can be BI-RADS 3, the follow-up interval can be increased to 1 year. Assuming 24 months of stability, the patient can revert to BI-RADS 2 or one can continue as BI-RADS 3 recommending imaging in 1 year assuming no need for biopsy. A finding can be upgraded to BI-RADS 4 or 5 or downgraded to BI-RADS 2 at any point along the follow-up. The timing of follow-up exams is the same for ultrasound and MRI.

Compliance with BI-RADS 3 recommendations is far from perfect. A recent study by Chung et al. found that 83.3\% complied with the first 6-month follow-up, decreasing over time to $75.9 \%$ at 12 months and $53.9 \%$ at 24 months [10]. A strong navigation program is needed to maximize compliance, but even with one's best efforts patients may not return for reasons beyond the radiologist's control including insurance issues especially for MRI follow-ups.

\section{BI-RADS 3 in Mammography}

BI-RADS 3 is not appropriate at screening mammography. After a complete diagnostic evaluation, classifying a mammographic finding as a BI-RADS 3 is highly predictive of benignity $[4,11,12]$ and allows for short interval follow-up rather than biopsy. While a BI-RADS 3 categorization allows for a decrease in the number of biopsies and their associated risks and costs, it should only be used to describe specific findings including a solitary group of round or punctate calcifications (Fig. 1), a non-calcified well-circumscribed solitary mass (Fig. 2), or a focal asymmetry (Fig. 3) without calcification or architectural distortion. [1, 13-15].

A single group of punctate or round calcifications may be classified as a BI-RADS 3 after appropriate evaluation with magnification views [1]. Additionally, calcifications suggestive of early fat necrosis (Fig. 4) in a patient who has undergone biopsy or trauma as well as calcifications that the radiologist believes are most likely vascular can be categorized as probably benign [13]. During follow-up, an increase in the number of calcifications that is not consistent with an evolving benign cause, or a change in calcification morphology causing them to appear more suspicious should prompt a biopsy recommendation [16]. For BI-RADS 3 to be properly used, the calcifications must be properly assessed. Amorphous calcifications, for example, carry a greater risk of malignancy and should not be assigned BI-RADS 3 [17].

A non-calcified solid mass that is round or oval with circumscribed margins can be classified as BI-RADS 3 [15]. If a mass has overlapping fibroglandular tissue obscuring the margins, it can be categorized as probably benign if at least $75 \%$ of the margins are circumscribed and no portion of the visualized margin is suspicious. This may require obtaining spot compression or magnification views $[15,18]$ or an ultrasound for further evaluation $[19,20]$. If a mass in this category demonstrates stability, it can be categorized as definitively benign and assessed as BIRADS $2[5,6]$. However, a mass with benign characteristics that demonstrates interval growth or a suspicious

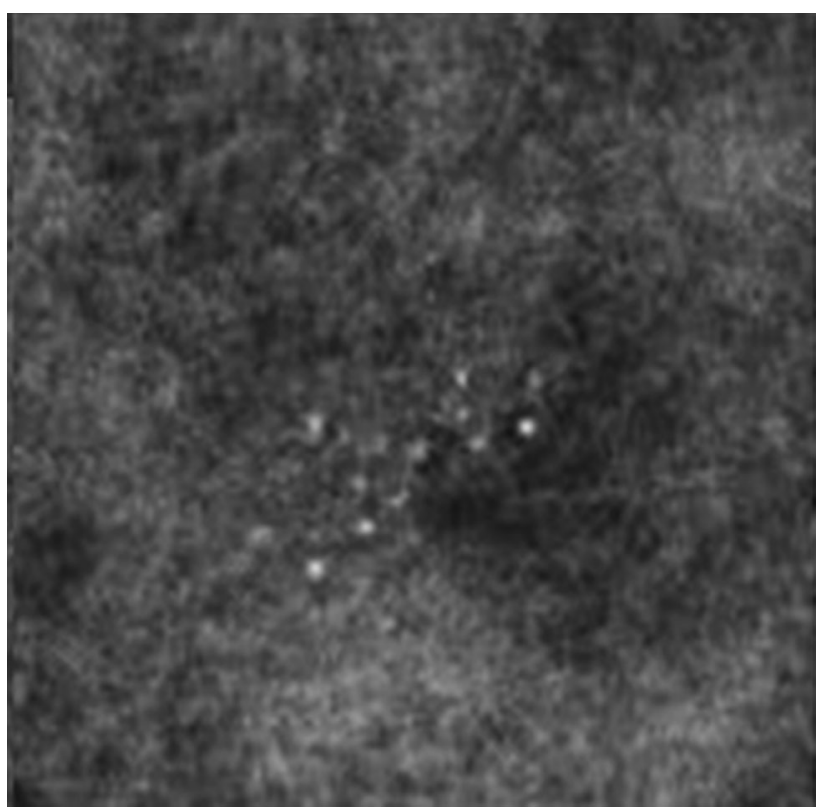

Fig. 1 Mammographic appearance of solitary group of round or punctate calcifications, which are appropriate for BI-RADS 3 
Fig. 2 Non-calcified circumscribed oval mass on mammography

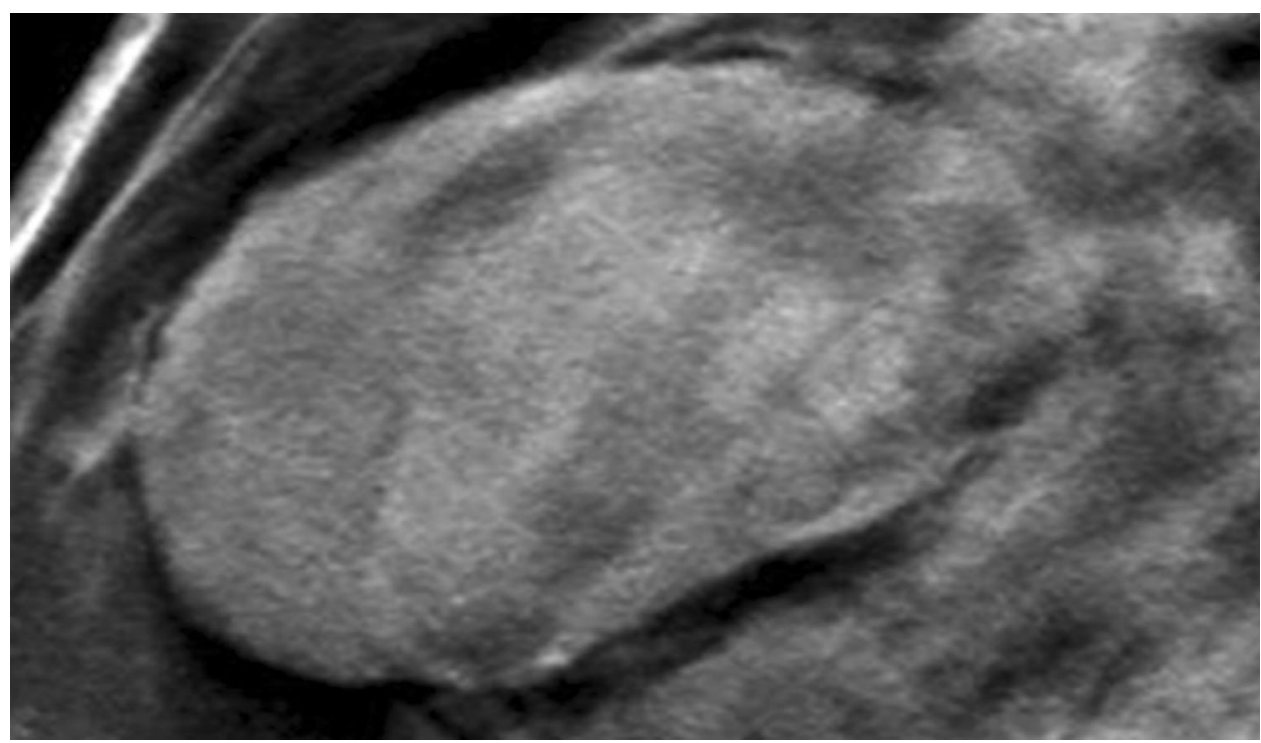

change in morphology cannot appropriately be considered a BI-RADS 3 and should be recommended for biopsy $[21,22 \bullet \bullet$.

A focal asymmetry is a density with concave borders, which is contained in a single quadrant and is seen on at least two mammographic projections [2]. It is often interspersed with fat and in the absence of calcification or architectural distortion it can be classified as probably benign if initially detected on a baseline examination [1]. However, if a focal asymmetry is new or increased in size it is not a true focal asymmetry, but rather a developing asymmetry and should be biopsied as developing asymmetries seen at screening have a $>12 \%$ chance of malignancy [23].

The BI-RADS atlas also provides some room for radiologist's discretion by allowing one to place findings in a BI-RADS 3 category if one's personal experience would allow one to justify the assessment. For example, in addition to calcifications that may be vascular or fat necrosis, an asymmetry or distortion thought to represent a postsurgical scar may be assigned BI-RADS 3 (Fig. 5). Also, if there are technical differences between exams that make it difficult to assess stability, one could assess a finding as BIRADS 3. This happened with the change from analog to digital and now happens with the change from full field digital to digital breast tomosynthesis (DBT) [2].

The BI-RADS 3 assessment should not be assigned at screening mammography; one reason is the relatively high rates of non-compliance with short interval follow-up recommendations [24, 25]. Omitting diagnostic workup could delay diagnosis of cancer at a lower stage and smaller size [26], potentially impacting treatment and/or prognosis. In contrast, unnecessary follow-up of a finding that could have been proven benign at diagnostic workup can increase overall cost and patient anxiety. For example, prompt workup of a mass seen on screening mammography may indicate that it is a benign cyst, and patient would not need short interval follow-up examinations.

Digital breast tomosynthesis (DBT) has impacted the use of BI-RADS 3 at diagnostic mammography. Raghu et al. found that over a 3-year time frame the use of BIRADS 3 at diagnostic mammography fell from 33.3 to $16.4 \%$ with no change in the percentage of BI-RADS 4 and 5 findings. Many of those who were previously placed into the probably benign assessment category are now given a normal report. This is in large part due to DBT's ability to assess focal asymmetries as normal tissue [27]. Similarly, McDonald et al. found that screening with DBT decreased the overall number of patients recommended for short interval follow-up by a mean of 2.4 women per 1000 , compared to screening with digital mammography [28••].

While the use of BI-RADS 3 in mammography continues to evolve, it has served as a paradigm for its implementation in ultrasound and MRI.

\section{Ultrasound BI-RADS 3}

Ultrasound is readily available, uses no ionizing radiation, and is well tolerated by patients. In women with dense breast tissue, supplemental breast ultrasound imaging can increase cancer detection rates by 2.3-4.6 per 1000 . However, in finding more cancers, supplemental ultrasound will also discover more benign masses that are not characteristically benign in appearance, increasing the number of biopsies and false-positive rates [29-34, 35•, 36, 37].

BI-RADS ultrasound descriptors have been shown to distinguish between malignant and benign masses with 
Fig. 3 Focal asymmetry without calcifications or architectural distortion

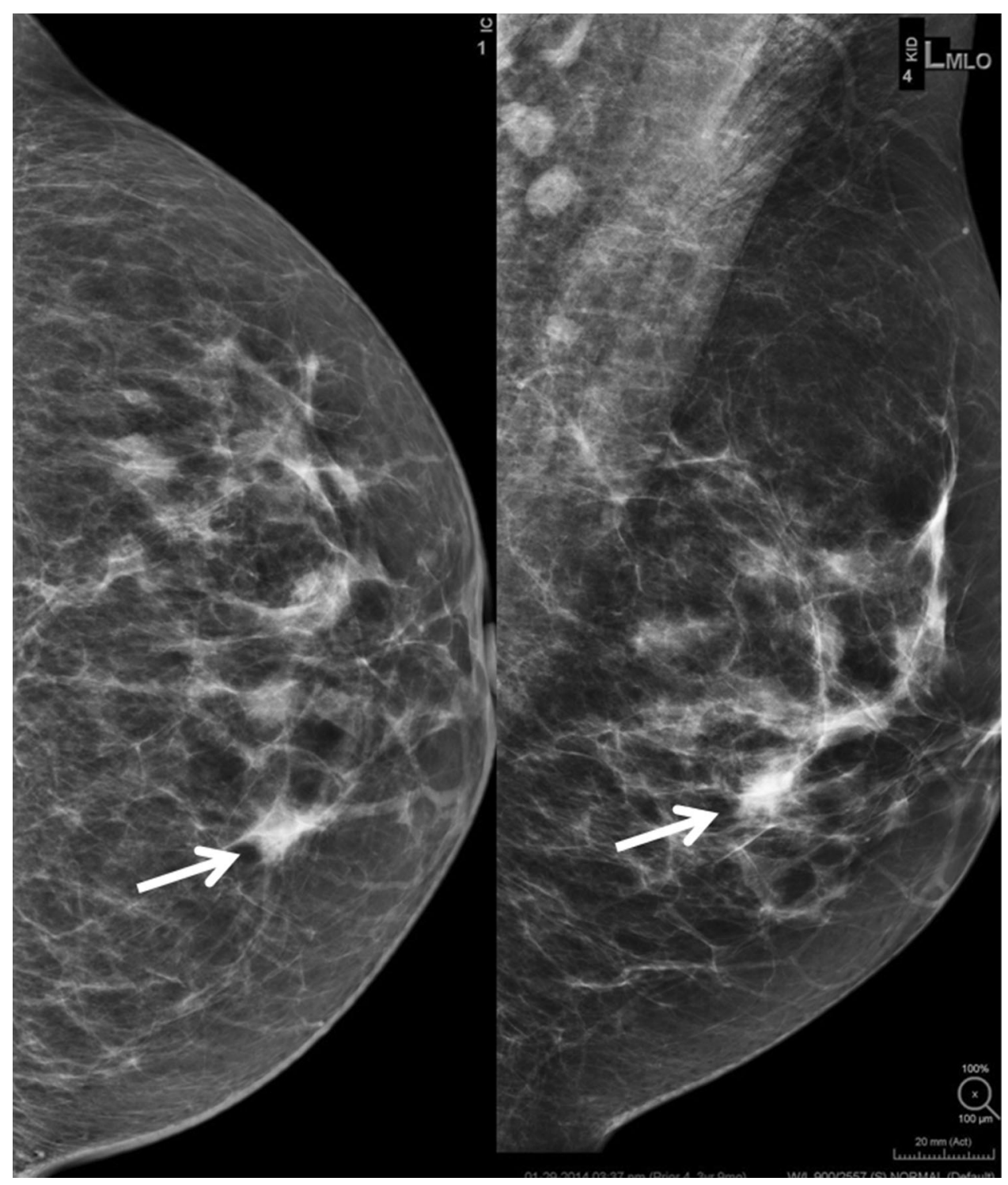

Fig. 4 Calcifications in a patient with a history of trauma, consistent with fat necrosis

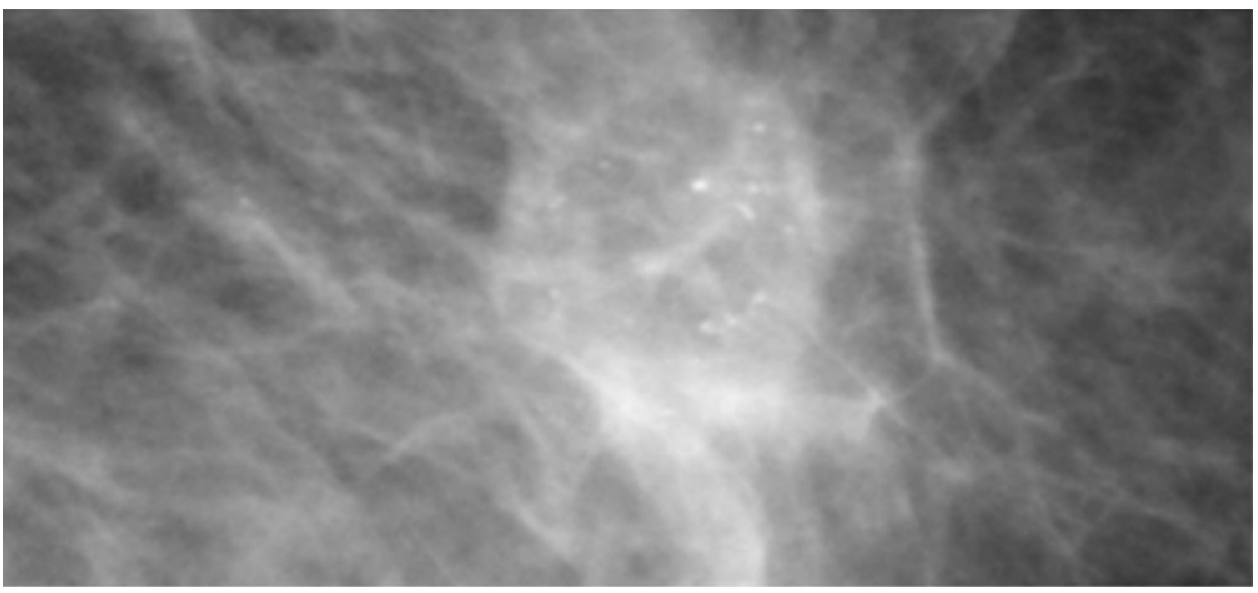

high positive and negative predictive values, respectively $[38,39]$. Sonographic masses that meet criteria for BIRADS 3, like their counterparts in mammography and
MRI, have a less than or equal to $2 \%$ likelihood of malignancy. This category reduces the number of falsepositive biopsies and justifies a period of watchful waiting, 
Fig. 5 Focal asymmetry with post-surgical architectural distortion seen on mammography and ultrasound in a patient with prior breast surgery. This was initially assessed as BI-RADS 3 but subsequent follow-up mammograms demonstrate long-term stability

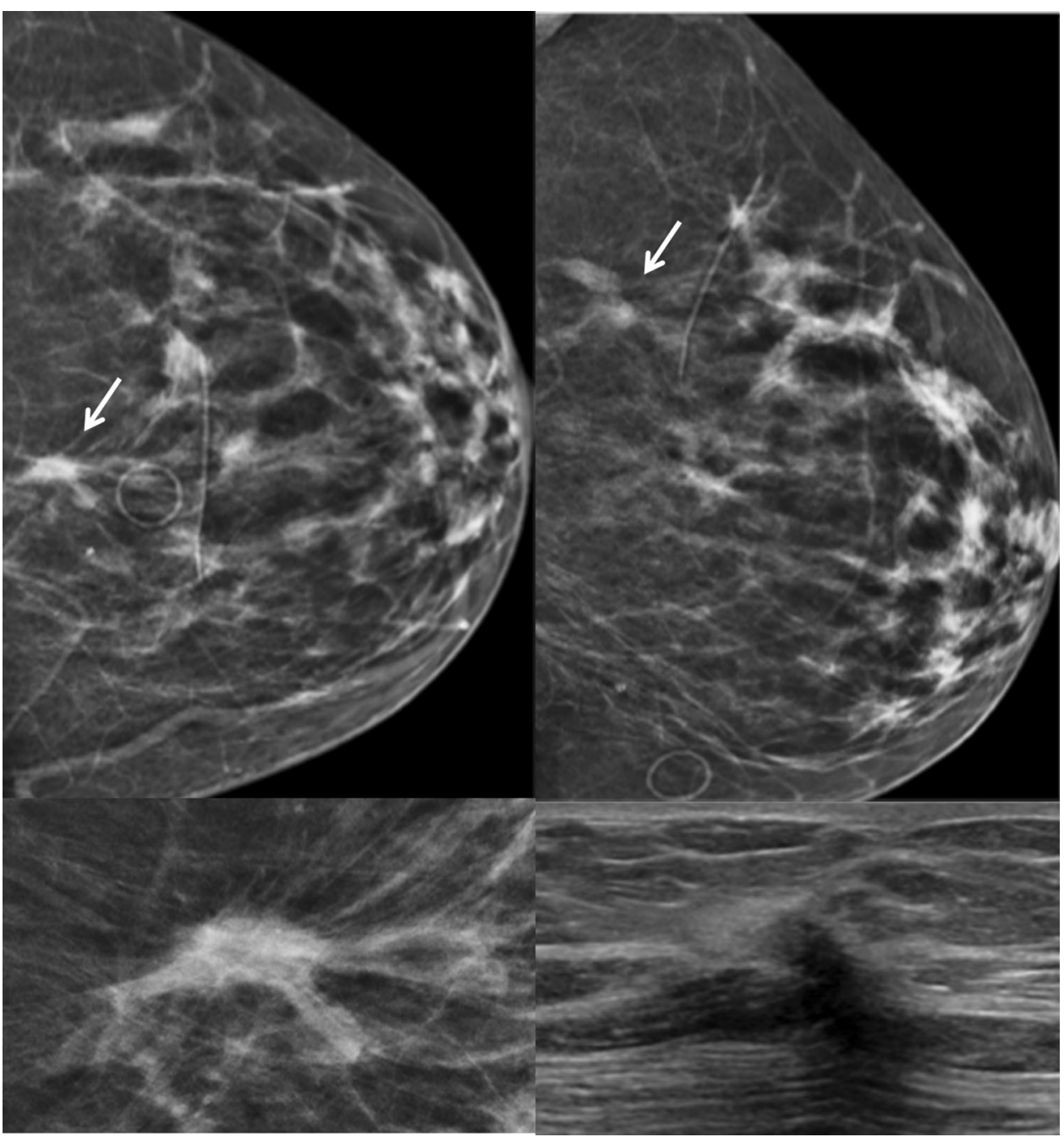

avoiding unnecessary workup when the likelihood of malignancy is very low [20, 40-42].

The characteristics that determine a BI-RADS 3 mass on ultrasound include benign features such as an oval shape, well-circumscribed margins, parallel orientation, echogenicity less than fat with no posterior features or minimal posterior acoustic enhancement [43]. Some masses that are commonly assessed as BI-RADS 3 include classic appearing fibroadenomas, an isolated complicated cyst or cluster of microcysts that is perhaps diagnostically challenging or new in a postmenopausal woman not on hormonal therapy (Fig. 6). Greenwood has shown that no cluster of microcysts was found to be malignant [44], confirming that BI-RADS 2 or 3 are appropriate assessments of the clustered microcysts. There are instances where the personal experience of the radiologist may warrant a shorter interval follow-up, which may not fall into the typical BI-RADS 3 follow-up interval. Such cases include fat necrosis or hematomas, where worrisome imaging appearance may not justify a full 6 -month wait time. Commonly, women do not remember trauma to the breast and therefore fat necrosis is often worked up and biopsied. However, when there is known breast trauma and a suspicion of fat necrosis or hematoma, a BI-RADS 3 assessment with a tailored shorter interval follow-up (4-8 weeks) may be considered (Fig. 7). Evolution of the fat necrosis or resolution of a hematoma over a short interval time will confirm the diagnosis. For example, a hematoma will likely transform from a hyperechoic heterogeneous mass to a hypoechoic smaller mass over weeks or months [22••, 39].

BI-RADS 3 is used for both palpable and non-palpable masses and can accurately predict benignity when combining clinical information with mammographic and ultrasound findings [40, 42, 45]. A mass may be categorized as BI-RADS 3 during handheld screening ultrasound - as opposed to screening mammography - as this modality is often read in real time and the findings can be detected and evaluated simultaneously as a combined screening and diagnostic exam. When a woman has 

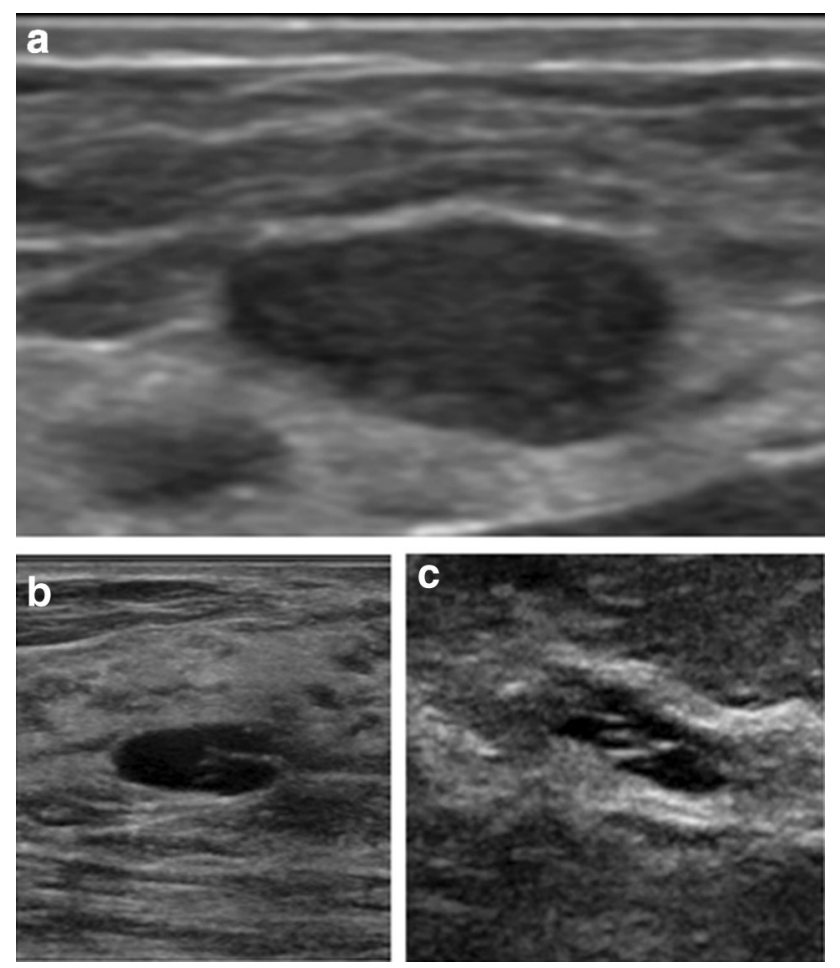

Fig. 6 a Typical appearance of a BI-RADS 3 oval mass, with circumscribed margins and parallel orientation. b Anechoic cyst with thin internal septations, assessed as BI-RADS 3. c Clusters of anechoic microcysts, assessed as BI-RADS 3

multiple bilateral masses, each mass should be evaluated separately and BI-RADS assigned to the most suspicious mass. If all masses are similar and there are more than 3 (with 1 on one side and 2 on the other), data from ACRIN 6666 indicate that they may be assigned BI-RADS 2 [46].

Similar to the mammographic BI-RADS 3 protocols, ultrasound BI-RADS 3 masses will typically undergo a 6-, 12-, and 24-month surveillance protocol to ensure stability and continued benign appearance. After 24 months of stability, the patient may return to routine screening. If during this surveillance period, the mass decreases in size or demonstrates resolution, it can be downgraded to a BIRADS 2. If during the surveillance period the mass grows in size or demonstrates suspicious qualities, then the BIRADS category may be upgraded to a biopsy recommendation. Significant interval increase in size of a mass, generally accepted as greater than $20 \%$, can supersede benign morphology of a mass and often warrants a biopsy [47]. Significant interval growth is concerning for pathologies such as mucinous, medullary, or papillary carcinomas (Fig. 8). A recent study by Jang et al. showed that the malignancy rate for enlarging or morphologically changed lesions was significantly higher than for stable lesions [48]. If the mass is indeterminate or has any suspicious characteristics, a BI-RADS 3 category should not be issued and biopsy should be pursued. In these cases, a wait period is not justified and may delay diagnosis of cancer.

Chae et al. demonstrated the radiologist's difficulty using BI-RADS 3 for breast ultrasound. In their experience, $14.6 \%$ of screening breast ultrasounds were read as BIRADS 3. When, however, they reinterpreted the ultrasound exams using ACRIN 6666 criteria, 19.3\% of cases had an assessment change. Of 225 patients who had a BI-RADS change, 213 were changed to BI-RADS 2 and 12 were upgraded to BI-RADS 4 [35॰]. Chae also found that the malignancy rate was higher for those with abnormal mammograms compared with those who had normal mammograms $(2.2 \%$ vs $0.4 \%)$ [35•]. This suggests an avenue for future research.

As ultrasound techniques continue to improve with higher-frequency linear transducers that increase spatial resolution, spatial compounding to improve margin analysis, tissue harmonic imaging that reduces near-field artifacts and intensifies posterior acoustic features, and more robust power Doppler, radiologists have the opportunity to increase their diagnostic confidence [44]. This may lead to further refinements of BI-RADS 3 ultrasound criteria.
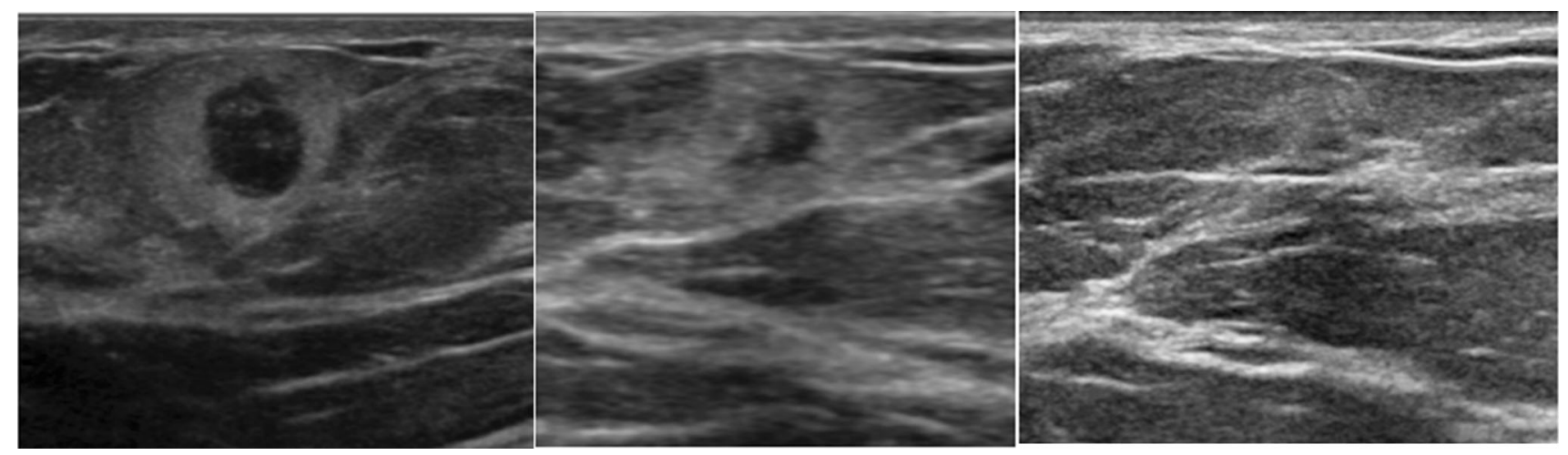

Fig. 7 Weeks 0, 6, and 11: suspected fat necrosis in a patient with known breast trauma. Short interval follow-up demonstrates decreasing size of the mass confirming the diagnosis 


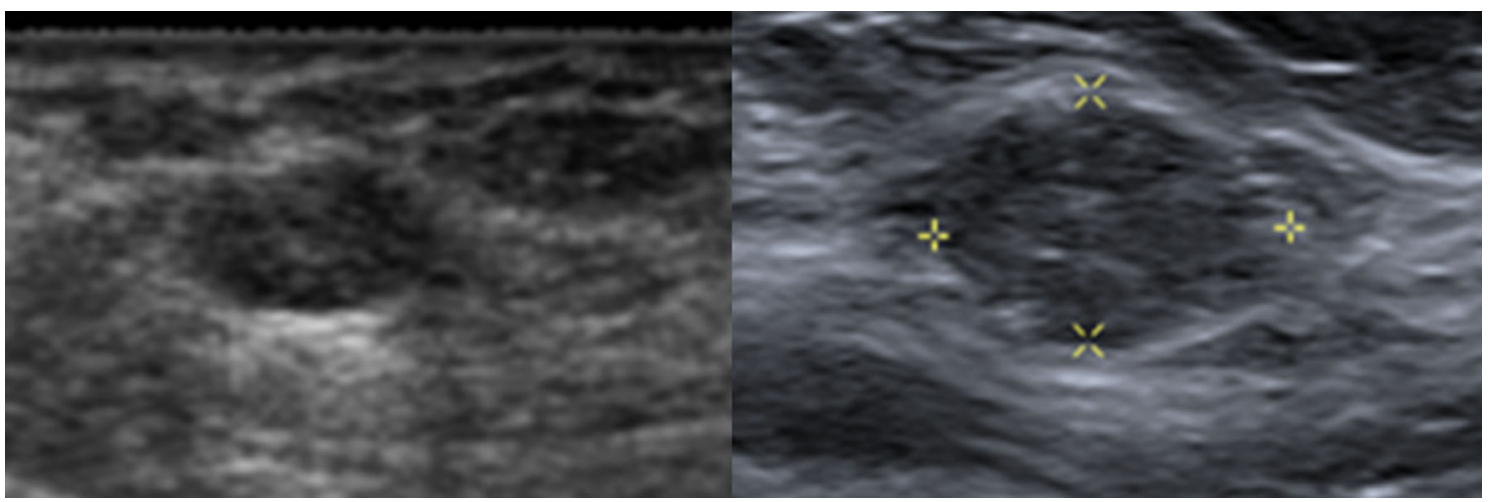

Fig. 8 Interval growth of a BI-RADS 3 mass. This solid oval mass on ultrasound was assessed as BI-RADS 3. However, patient was lost to follow up and did not return for 2 years. The mass was biopsied at 2-year follow-up due to interval growth yielding mucinous carcinoma

\section{BI-RADS 3 in Breast MRI}

BI-RADS 3 for MRI was adapted from mammography [2]. While there are well-established criteria for the use of BIRADS category 3 for mammography, similar criteria have not been established for MRI. Several studies have demonstrated that lesions assigned to BI-RADS 3 category have a $\leq 2 \%$ malignancy rate. Association with specific BI-RADS lesion descriptors, however, could not be established $[49,50]$. The majority of studies on the frequency of BI-RADS 3 report that between 6 and 12\% of examinations are assessed as BI-RADS 3 (Table 1).

There are unique features to MRI BI-RADS 3 assessments. The population undergoing screening MRI is at

Table 1 Frequency of MR imaging BI-RADS 3 assessment category and cancer yield

\begin{tabular}{|c|c|c|c|c|c|}
\hline Reference & Type & Study population & $\begin{array}{l}\text { Probably benign } \\
\text { examinations } \\
\text { (number [\%]) }\end{array}$ & $\begin{array}{l}\text { Probably benign } \\
\text { patients } \\
\text { (number [\%]) }\end{array}$ & $\begin{array}{l}\text { Cancer yield } \\
\text { (number [\%]) }\end{array}$ \\
\hline Kuhl et al. [73] & Prospective & High risk & 45/363 (12.4) & 44/192 (22.9) & $1 / 44(2.3)$ \\
\hline Liberman et al. [74] & Retrospective & High risk & $89 / 367(24.2)$ & $89 / 367(24.2)$ & 9/89 (10.1) \\
\hline Kriege et al. [75] & Prospective & High risk & $275 / 4169(6.6)$ & NR/1909 & $3 / 275(1.1)$ \\
\hline Hartman et al. [76] & Prospective & High risk & $19 / 75(25)$ & $14 / 41(34.1)$ & $0 / 14(0.0)$ \\
\hline Sadowski and Kelcz [77] & Retrospective & BI-RADS 0 mammogram & NR & 79/473 (16.7) & $4 / 68(6)$ \\
\hline Kuhl et al. [78] & Prospective & High risk & $167 / 1452(11.5)$ & $\mathrm{NR} / 529$ & NR \\
\hline Eby et al. [79] & Retrospective & $\begin{array}{l}\text { High risk, extent of disease, } \\
\text { problem solving }\end{array}$ & $160 / 809(20)$ & $160 / 678(23.6)$ & $1 / 160(0.6)$ \\
\hline Eby et al. [59] & Retrospective & $\begin{array}{l}\text { High risk, extent of disease, } \\
\text { problem solving }\end{array}$ & $260 / 2569(10.1)$ & 236/1735 (13.6) & $2 / 236(0.9)$ \\
\hline Weinstein et al. [80] & Prospective & Known contralateral cancer & 106/969 (10.9) & 106/969 (10.9) & $1 / 106(0.9)$ \\
\hline Hauth et al. [81] & Retrospective & $\begin{array}{l}\text { High risk, extent of disease, } \\
\text { problem solving }\end{array}$ & $44 / 698(6.3)$ & 44/698 (6.3) & $1 / 56(1.8)$ \\
\hline Mahoney et al., 2012 [50] & Prospective & Known contralateral cancer & 106/969 (10.9) & 106/969 (10.9) & 1/101 (0.9) \\
\hline Lourenco et al. [82] & Retrospective & $\begin{array}{l}\text { High risk, abnormal imaging, } \\
\text { extent of disease, clinical } \\
\text { symptom }\end{array}$ & $348 / 4370(8)$ & $\mathrm{NR} / 345$ & $5 / 345$ (1.4) \\
\hline Bahrs et al. [58] & Retrospective & $\begin{array}{l}\text { High risk, extent of disease, } \\
\text { problem solving }\end{array}$ & $182 / 666(27.3)$ & 117/NR (17.6) & 3/163 (1.8) \\
\hline Spick et al. [54] & Retrospective & $\begin{array}{l}\text { Not high risk, no history of } \\
\text { breast cancer }\end{array}$ & $108 / 1265(8.5)$ & $108 / 1265(8.5)$ & 1/108 (0.9) \\
\hline Grimm et al. $[83 \bullet \bullet]$ & Retrospective & $\begin{array}{l}\text { High risk, extent of disease, } \\
\text { problem solving, clinical } \\
\text { symptoms }\end{array}$ & 282/4279 (6.6) & 265/3131 (8.4) & $12 / 280(4.3)$ \\
\hline Chikarmane et al. [84] & Retrospective & High risk, diagnostic purposes & NR/5778 & 483/3360 (14.3) & $11 / 435(2.5)$ \\
\hline
\end{tabular}

$N R$ not reported 

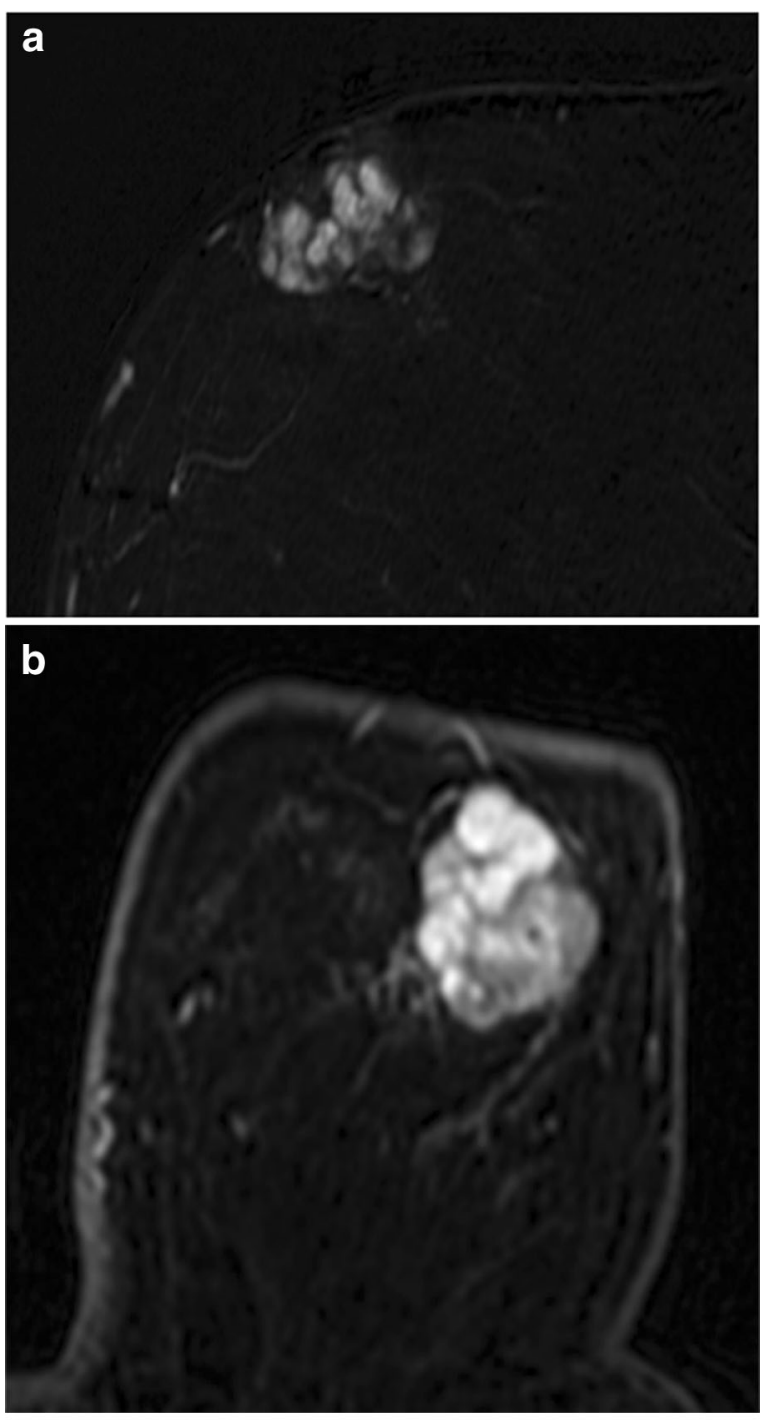

C

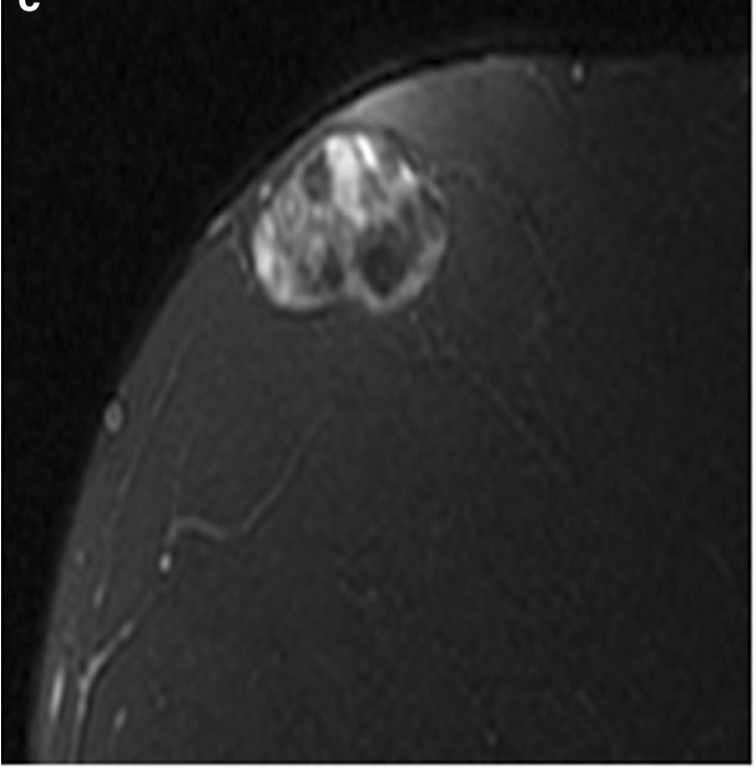

४Fig. 9 Fibroadenoma. Postcontrast subtraction T1-weighted sagittal (a) and axial (b) images show a 3.6-cm oval mass with circumscribed margins and dark internal septations. On c fat-saturated T2-weighted image, it demonstrated high signal intensity and is most consistent with a fibroadenoma. If this mass was an incidental finding on baseline MRI, a BI-RADS 3 assessment would be appropriate
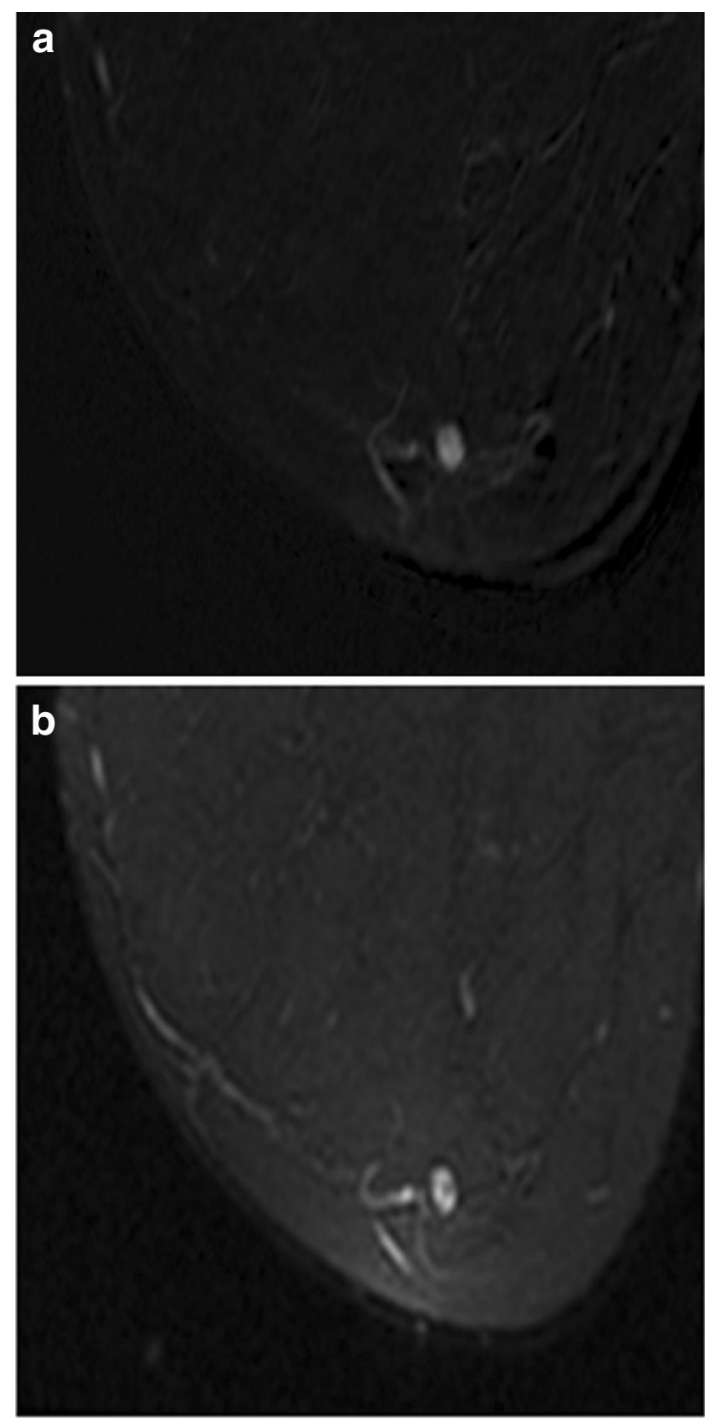

Fig. 10 Mass with oval shape and circumscribed margins. a Postcontrast subtraction T1-weighted image shows a $0.6-\mathrm{cm}$ oval-shaped mass with circumscribed margins and homogeneous internal enhancement, which demonstrated high signal on T2-weighted sequence (b) and a BI-RAD 3 assessment was given. 6-month follow-up MRI showed that this mass was stable and is likely an intramammary lymph node. This example shows that BI-RADS 3 assessment is appropriate for masses with an oval shape and circumscribed margins on baseline examination

higher lifetime risk for developing breast cancer than those undergoing screening mammography. Also, the clinical indication for performing MRI is different from mammography. Patients undergoing MRI because of a known 
Fig. 11 Focus with the absence of high signal on $\mathrm{T} 2$ sequence. a Postcontrast subtraction T1weighted image shows a unique $0.4-\mathrm{cm}$ focus with washout delayed kinetics (b) and the absence of high signal on fatsaturated T2-weighted image (c). Because this focus was new, it was assessed as probably benign, BI-RADS 3. Follow-up examination 6 months later showed increase in size of the focus; therefore, biopsy was recommended. MRI-guided wire localization was performed of this focus and surgery yielded invasive ductal carcinoma. For foci with washout kinetics and the absence of high T2 signal, biopsy should be considered
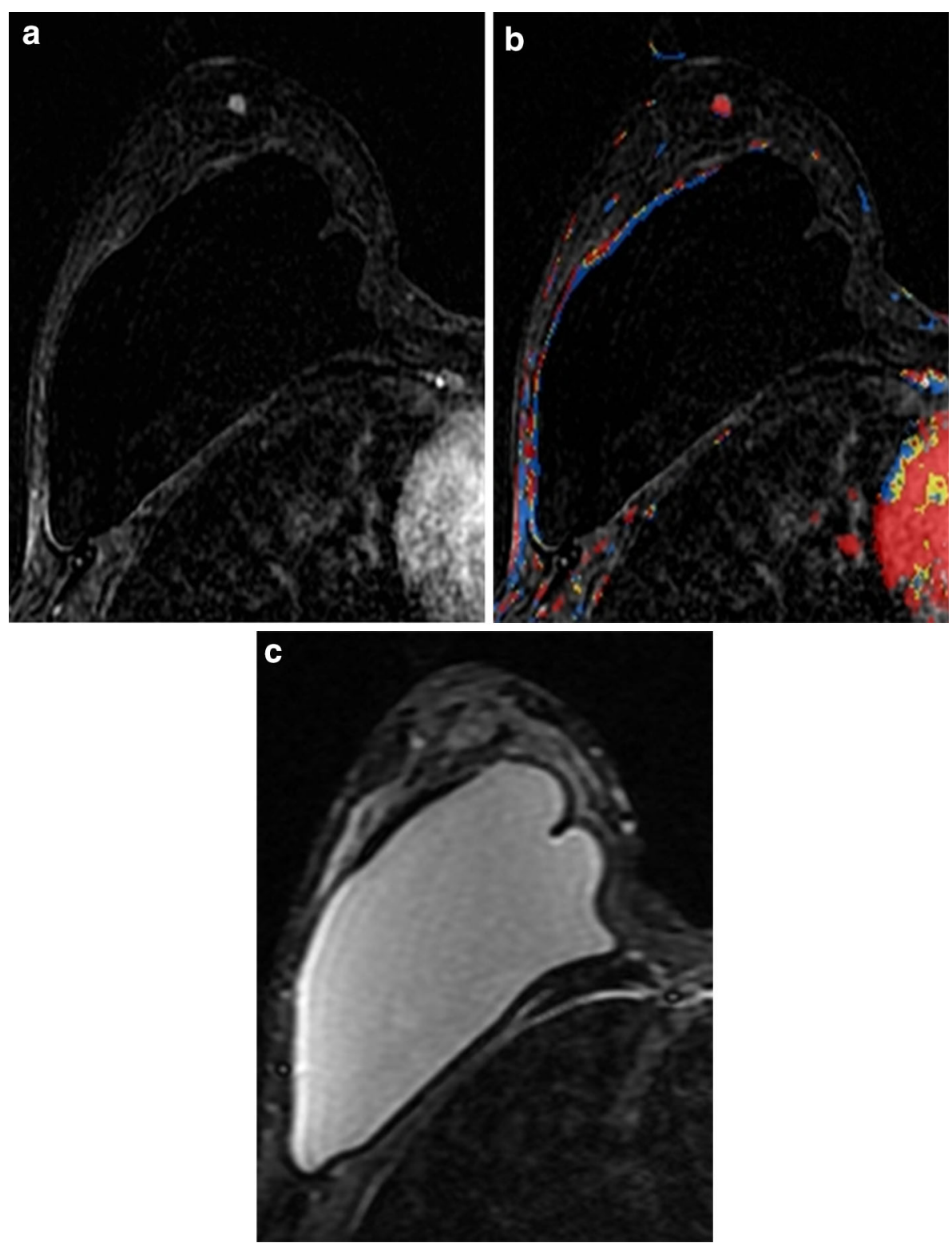

breast cancer to evaluate the extent of disease have a higher frequency of additional areas of cancer, thus raising the suspicion of findings that would otherwise be less worrisome $[51,52,53 \bullet \cdot]$. Furthermore, the utility of short-term follow-up for a patient who is about to begin breast cancer treatment is disputable. In addition to the actual finding characteristics, a patient's breast cancer risk and planned breast cancer treatment should be collectively assessed when MRI findings are assigned BI-RADS 3.

A mass is a space-occupying three-dimensional lesion, which has a defined shape, margin, and internal enhancement characteristics. The most appropriate and common use of BI-RADS 3 assessment is for a round- or ovalshaped mass with circumscribed margins and hyperintense T2 signal, which has either homogeneous enhancement or dark internal septations on a baseline examination. A mass meeting these criteria is most likely an intramammary lymph node or fibroadenoma. (Fig. 9). Therefore, a mass with a round or oval shape, circumscribed margins, and persistent or plateau kinetic curve should be assigned BIRADS 3 on baseline examination [54] (Fig. 10). Although increased $\mathrm{T} 2$ signal is most often associated with benign masses, it has been reported in subsets of breast cancers, particularly of the mucinous and papillary subtype $[55,56]$. When mass features are studied, the single most predictive feature for malignancy is the margin $[25,57]$ not the $\mathrm{T} 2$ signal.

Foci represent up to $41-48 \%$ of BI-RADS 3 lesions $[58,59]$, but are rarely malignant. A focus is a unique enhancing dot, usually less than $5 \mathrm{~mm}$, which is too small 

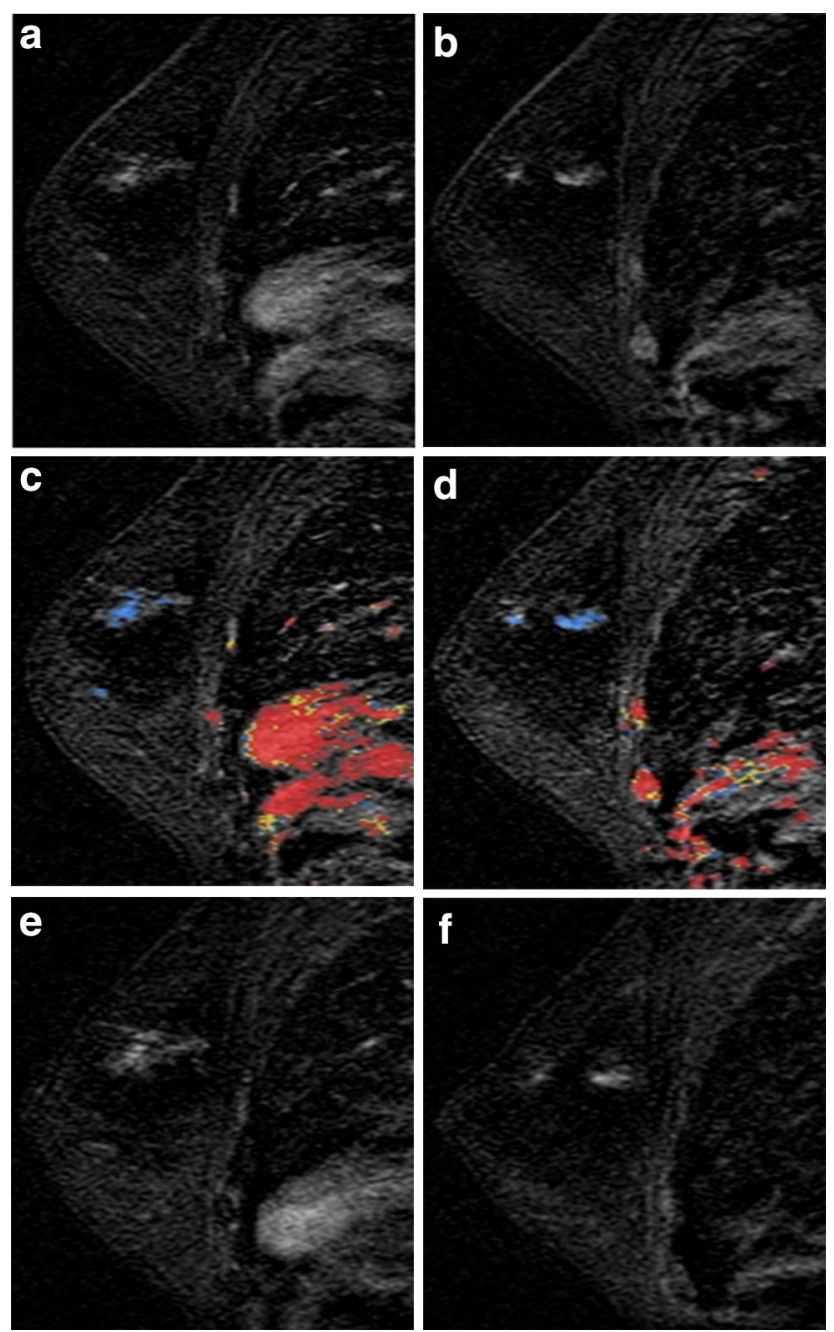

Fig. 12 Multiple regions of non-mass enhancement (NME) in the same breast. Postcontrast subtraction T1-weighted images a, b show multiple regions of NME, which are new but demonstrated persistent kinetics $(\mathbf{c}, \mathbf{d})$. These were assessed as probably benign, given the multiplicity, and were assumed to be transient enhancement related to hormonal status in this premenopausal woman. Follow-up exam 6 months later demonstrates slight increase in degree of enhancement $(\mathbf{e}, \mathbf{f})$; therefore, MRI-guided biopsy was recommended and yielded ductal carcinoma in situ (DCIS). Patient elected mastectomy yielding diffuse DCIS, no invasive component. New areas of NME should raise suspicion and biopsy should be considered

to further characterize. Although the vast majority of foci are benign, new or enlarging foci should raise suspicion and prompt either short-term follow-up or biopsy [58] (Fig. 11).

A study by Eby et al. found that all foci that demonstrated persistent kinetics on delayed phase enhancement were benign, suggesting that all persistent foci can appropriately be assigned BI-RADS 2 [59]. There is, however, conflicting data on the utility of kinetic information in assessing foci. A retrospective study of 111 patients with 136 foci by Ha et al. showed that kinetics were not useful in distinguishing benign from malignant foci [60]. Ha et al. demonstrated a malignancy rate of 2.9\% (4 of 136 foci); the predictors of malignancy were an absence of high T2 signal intensity and a focus that was either new or increased in size.

There are limited data to support the use of BI-RADS 3 for non-mass enhancement (NME).

Non-mass enhancement is defined as enhancement that is not a mass and whose internal enhancement characteristics are unique from background parenchymal enhancement (BPE). A study by Schnall et al. showed that distribution was the most predictive of diagnosis in NME [57]. Additional studies have demonstrated that NME with a linear or segmental distribution requires biopsy because these descriptors not only have a greater than $2 \%$ malignancy rate but were most predictive of malignancy $[50,61]$.

Spick et al. report that BI-RADS 3 may be assigned if the NME is either focal or regional in distribution and the internal enhancement pattern is homogeneous with either persistent or plateau enhancement kinetic curve [54]. Regional, multiple regions, and diffuse distribution patterns were associated with the lowest probability of cancer [50]. It would therefore be acceptable to use BI-RADS 3 for NME with a focal or regional distribution, homogeneous internal enhancement on a baseline examination. However, if there is new focal or regional distribution of NME, suspicion should be raised and a biopsy would be appropriate (Fig. 12).

The 5th edition of the BI-RADS Atlas recommends an MRI short-term follow-up interval identical to that recommended for mammography [2]. Unique to MRI are issues concerning the patient who had an MRI-guided biopsy yielding benign concordant pathology. In this setting, a short-term follow-up MRI is appropriate in order to confirm adequate sampling of the targeted lesion. It is suggested that the most effective timing to perform this follow-up is 6 months after the biopsy [62]. MRI after benign concordant MRI-guided biopsy has shown that $8-12 \%$ of targeted lesions were inadequately sampled and, of those inadequately sampled, malignancy was ultimately diagnosed in $14-18 \%$ with a false-negative rate of MRIguided biopsy of 2.5\% [63]. Although no study has addressed, the significance of lesion stability 6 months following MRI-guided biopsy, the possibility of a missed target should be entertained (Fig. 13). Cancers, which were missed on MRI-guided biopsy, usually do not demonstrate appreciable change in size sooner than 6 months [64].

While there are no established criteria for the use of BIRADS 3 for breast MRI, there is increasing experience determining which lesions would be appropriate for BIRADS 3. An incidental round- or oval-shaped mass with circumscribed margins and hyperintense T2 signal, which 
Fig. 13 Missed MRI-guided biopsy with follow-up demonstrating cancer. a Postcontrast subtraction T1weighted image shows a $1.2-\mathrm{cm}$ non-mass enhancement (NME) with focal distribution, heterogeneous internal enhancement, and $\mathbf{b}$ washout kinetics (arrow), which was suspicious and assessed as BIRADS 4. MRI-guided biopsy was performed yielding fibrocystic changes and a 6-month follow-up MRI was recommended. At 6-month follow-up, c postcontrast subtraction T1-weighted image shows persistence of the NME and washout kinetics (d). Postcontrast T1-weighted image (e) shows that the susceptibility artifact from the biopsy marker clip is located posterior to the focal NME, which was unchanged in size and appearance suggesting that the NME was not biopsied. Surgical excision yielded carcinoma in situ
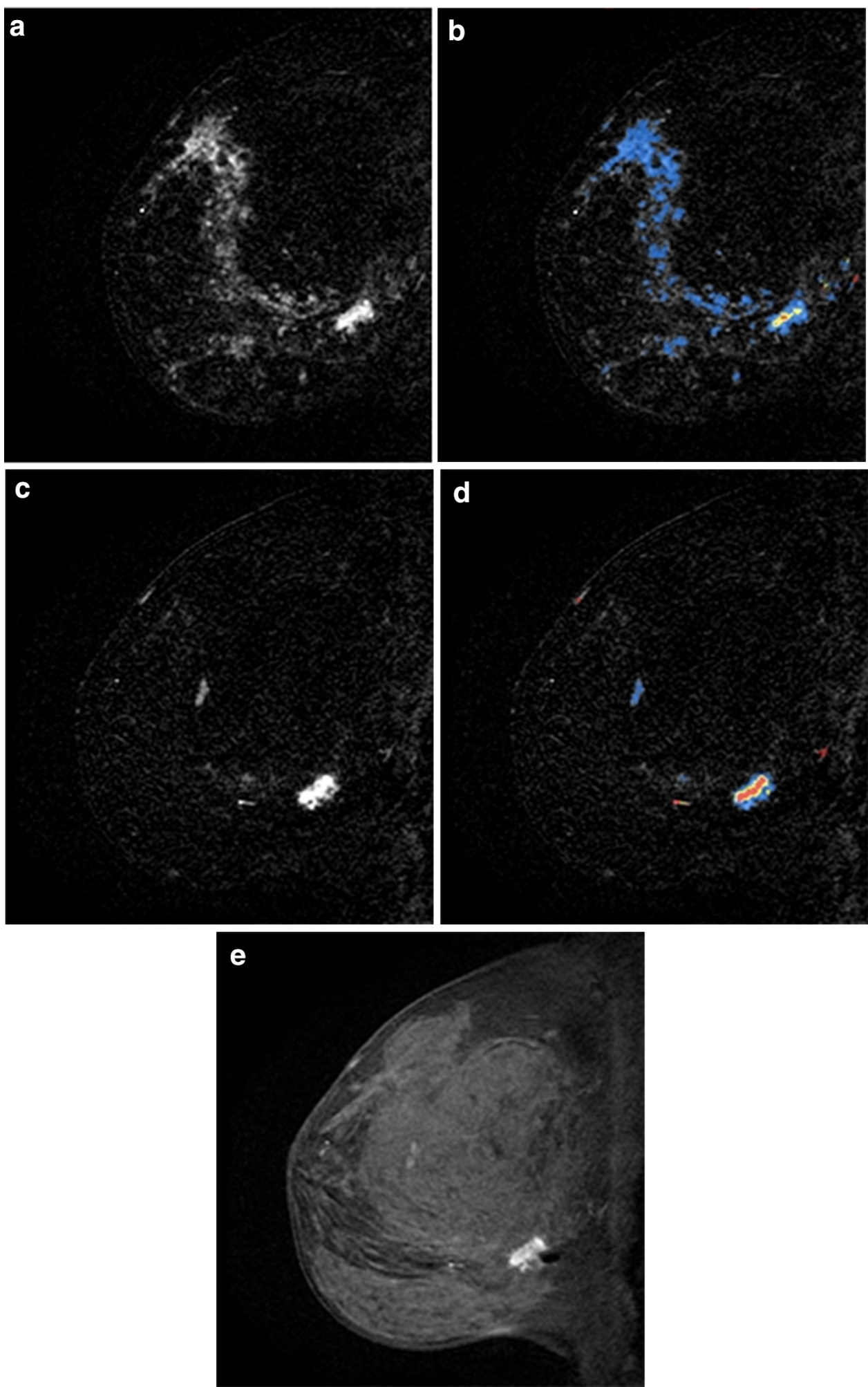

has either homogeneous enhancement or dark internal septations on a baseline examination is an appropriate use of BI-RADS 3. It also would be reasonable to assign a focus with washout kinetics into the BI-RADS 3 category on baseline examination. Short-term follow-up can be recommended for homogeneous NME with a focal, regional or multiple regions distribution on baseline examination. When determining whether a BI-RADS 3 assessment would be appropriate, it is imperative to consider the 
patient's breast cancer risk and potential planned breast cancer treatment.

\section{Future Directions in the BI-RADS 3 Assessment Category}

Just as the clinical setting is important in MRI, work by Burnside et al. has shown that there are risk factors that should give one pause before assigning a BI-RADS 3. A logistic regression model that included age, personal breast cancer history, family breast cancer history, breast density, and mammogram features was applied to almost 5000 mammograms that had been interpreted as BI-RADS 3. A greater than $2 \%$ diagnosis of malignancy occurred in those patients who had a personal history of breast cancer and were over 50 years old who were placed into the BI-RADS 3 category at diagnostic mammography [65]. This work highlights the importance of looking beyond the images before deeming a finding probably benign.

Linda et al. attempted to decrease the numbers of BIRADS 3 assessments of calcifications by adding a contemporaneous MRI. They sought to determine if a normal MRI would indicate that the BI-RADS 3 calcifications were indeed benign and the patient could be returned to annual screening. Unfortunately, there was no statistically significant difference in the ultimate malignancy rate of those with positive and negative MRI exams. Thus, MRI cannot be used to exclude malignancy in the case of BIRADS 3 calcifications [66].

Elastography was evaluated by Cho et al. to determine its ability to upgrade or downgrade BI-RADS 3 masses. In their study, 276 BI-RADS 3 masses were evaluated with elastography. No invasive cancers were included in this cohort. 166 had negative elastograms with 1 of those patients having DCIS. If the negative elastograms were used to change the assessment from BI-RADS 3 to BIRADS 2, in this cohort the malignancy rate would have changed from 1 to $1.8 \%$ [67]. Larger studies that include some invasive cancers in the BI-RADS 3 group are needed to validate these results.

Future directions in MRI that have been studied include the use of diffusion weighted imaging (DWI) to determine its utility in the BI-RADS 3 mass. When Dijkstra et al. added DWI with intravoxel incoherent motion to standard MRI, the specificity increased from 30.4 to $56.6 \%$ with a negative predictive value of $92.9 \%$. This study is limited by including only large lesions, a very specific technique and small sample size, but points to the possibility of further technical refinements in MRI that could impact patient care [68].

BI-RADS 3 will continue to evolve as we more to making its use ever more evidence based and less intuitive
[69]. Continued research is needed to allow the practicing radiologist to properly and consistently use BI-RADS 3 across all breast imaging modalities including the less commonly available contrast-enhanced mammography [70] and molecular breast imaging [71, 72].

\section{Conclusion}

BI-RADS 3, probably benign, is a challenging assessment category. While its use in MRI is evolving, there are specific criteria for the designation of a mammographic or ultrasound finding as BI-RADS 3. Additionally, one's personal experience may allow other findings to fall into the probably benign category. Using the criteria outlined in the BI-RADS atlas and careful attention to patient characteristics such as age and ability and/or willingness to return for multiple follow-up visits will optimize the use of this most taxing and dynamic BI-RADS assessment category.

\section{Compliance with Ethical Guidelines}

Conflict of interest Karen A. Lee, Nishi Talati, Rebecca Oudsema, and Sharon Steinberger each declare no potential conflicts of interest.

Human and Animal Rights and Informed Consent This article does not contain any studies with human or animal subjects performed by any of the authors.

Open Access This article is distributed under the terms of the Creative Commons Attribution 4.0 International License (http:// creativecommons.org/licenses/by/4.0/), which permits unrestricted use, distribution, and reproduction in any medium, provided you give appropriate credit to the original author(s) and the source, provide a link to the Creative Commons license, and indicate if changes were made.

\section{References}

Recently published papers of particular interest have been highlighted as:

- Of importance

- Of major importance

1. Sickles EA. Periodic mammographic follow-up of probably benign lesions: results in 3,184 consecutive cases. Radiology. 1991;179(2):463-8.

2. American College of Radiology. Breast imaging reporting and data system (BI-RADS) 5. Reston: American College of Radiology; 2013.

3. D'Orsi C, Bassett L, Berg W, et al. Breast imaging reporting and data system: ACR BI-RADS—breast imaging Atlas. In: D'Orsi C, Mendelson E, Ikeda D, et al., editors. BI-RADS: mammography. 4th ed. Reston: American College of Radiology; 2003. p. 7-201. 
4. Helvie MA, Pennes DR, Rebner M, Adler DD. Mammographic follow-up of low-suspicion lesions: compliance rate and diagnostic yield. Radiology. 1991;178(1):155-8.

5. Vizcaíno I, Gadea L, Andreo L, Salas D, Ruiz-Perales F, Cuevas $\mathrm{D}$, et al. Short-term follow-up results in 795 nonpalpable probably benign lesions detected at screening mammography. Radiology. 2001;219(2):475-83.

6. Varas X, Leborgne F, Leborgne JH. Nonpalpable, probably benign lesions: role of follow-up mammography. Radiology. 1992;184(2):409-14.

7. Michaels AY, Chung CSW, Frost EP, Birdwell RL, Giess CS. Interobserver variability in upgraded and non-upgraded BI-RADS 3 lesions. Clin Radiol. 2017;72(8):694.e1-6.

8. Grimm LJ, Anderson AL, Baker JA, Johnson KS, Walsh R, Yoon $\mathrm{SC}$, et al. Interobserver variability between breast imagers using the fifth edition of the BI-RADS MRI Lexicon. AJR. 2015;204(5):1120-4.

9. Ortiz-Perez T, Trevino EJ, Sepulveda KA, Hilsenbeck SG, Wang T, Sedgwick EL. Does formal instruction about the BI-RADS ultrasound lexicon result in improved appropriate use of the lexicon? AJR. 2013;201(2):456-61.

10. Chung CS, Giess CS, Gombos EC, Frost EP, Yeh ED, Raza S, et al. Patient compliance and diagnostic yield of 18-month unilateral follow-up in surveillance of probably benign mammographic lesions. AJR. 2014;202(4):922-7.

11. Orel SG, Kay N, Reynolds C, Sullivan DC. BI-RADS categorization as a predictor of malignancy. Radiology. 1999;211(3):845-50.

12. Varas X, Leborgne JH, Leborgne F, Mezzera J, Jaumandreu S. Revisiting the mammographic follow-up of BI-RADS category 3 lesions. AJR. 2002;179(3):691-5.

13. Sickles EA. Probably benign breast lesions: when should followup be recommended and what is the optimal follow-up protocol? Radiology. 1999;213(1):11-4.

14. Adler DD, Helvie MA, Ikeda DM. Nonpalpable, probably benign breast lesions: follow-up strategies after initial detection on mammography. AJR. 1990;155(6):1195-201.

15. Leung JW, Sickles EA. The probably benign assessment. Radiol Clin North Am. 2007;45(5):773-89 (vi).

16. Lev-Toaff AS, Feig SA, Saitas VL, Finkel GC, Schwartz GF. Stability of malignant breast microcalcifications. Radiology. 1994;192(1):153-6.

17. Berg WA, Arnoldus CL, Teferra E, Bhargavan M. Biopsy of amorphous breast calcifications: pathologic outcome and yield at stereotactic biopsy. Radiology. 2001;221(2):495-503.

18. Sickles EA. Breast masses: mammographic evaluation. Radiology. 1989;173(2):297-303.

19. Bassett LW. Imaging of breast masses. Radiol Clin North Am. 2000;38(4):669-91 (vii-viii).

20. Graf O, Helbich TH, Hopf G, Graf C, Sickles EA. Probably benign breast masses at US: is follow-up an acceptable alternative to biopsy? Radiology. 2007;244(1):87-93.

21. Majid AS, de Paredes ES, Doherty RD, Sharma NR, Salvador X. Missed breast carcinoma: pitfalls and pearls. Radiographics. 2003;23(4):881-95.

22. • Michaels AY, Birdwell RL, Chung CS, Frost EP, Giess CS. Assessment and management of challenging BI-RADS category 3 mammographic lesions. Radiographics. 2016;36(5):1261-72. If a benign-appearing solid mass grows, the interval change supersedes benign morphology and biopsy is warranted despite probably benign features. Although interval change is a key feature of malignancy, many benign lesions also change and therefore, use of prior imaging and obtaining an accurate clinical history is important.
23. Leung JW, Sickles EA. Developing asymmetry identified on mammography: correlation with imaging outcome and pathologic findings. AJR. 2007;188(3):667-75.

24. Baum JK, Hanna LG, Acharyya S, Mahoney MC, Conant EF, Bassett LW, et al. Use of BI-RADS 3-probably benign category in the American College of Radiology imaging network digital mammographic imaging screening trial. Radiology. 2011;260(1):61-7.

25. Duijm LE, Zaat JO, Guit GL. Nonpalpable, probably benign breast lesions in general practice: the role of follow-up mammography. Br J Gen Pract. 1998;48(432):1421-3.

26. Duijm LE, Groenewoud JH, Jansen FH, Fracheboud J, van Beek M, de Koning HJ. Mammography screening in the Netherlands: delay in the diagnosis of breast cancer after breast cancer screening. Br J Cancer. 2004;91(10):1795-9.

27. Raghu M, Durand MA, Andrejeva L, Goehler A, Michalski MH, Geisel JL, et al. Tomosynthesis in the diagnostic setting: changing rates of BI-RADS final assessment over time. Radiology. 2016;281(1):54-61.

28. - McDonald ES, McCarthy AM, Weinstein SP, Schnall MD, Conant EF. BI-RADS category 3 comparison: probably benign category after recall from screening before and after implementation of digital breast tomosynthesis. Radiology. 2017:162837. Screening with digital breast tomosynthesis decreased the overall number of patients recommended for short interval follow-up by a mean of 2.4 women per 1000, compared to screening with digital mammography.

29. Destounis S, Arieno A, Morgan R. New York state breast density mandate: follow-up data with screening sonography. J Ultrasound Med. 2017;36:2511-7.

30. Hooley RJ, Greenberg KL, Stackhouse RM, Geisel JL, Butler RS, Philpotts LE. Screening US in patients with mammographically dense breasts: initial experience with Connecticut Public Act 09-41. Radiology. 2012;265(1):59-69.

31. Weigert JM. The Connecticut experiment; the third installment: 4 years of screening women with dense breasts with bilateral ultrasound. Breast J. 2017;23(1):34-9.

32. Weigert J, Steenbergen S. The connecticut experiment: the role of ultrasound in the screening of women with dense breasts. Breast J. 2012;18(6):517-22.

33. Weigert JM, Bertrand ML, Lanzkowsky L, Stern LH, Kieper DA. Results of a multicenter patient registry to determine the clinical impact of breast-specific gamma imaging, a molecular breast imaging technique. AJR. 2012;198(1):W69-75.

34. Berg WA, Blume JD, Cormack JB, Mendelson EB, Lehrer D, Bohm-Velez M, et al. Combined screening with ultrasound and mammography vs mammography alone in women at elevated risk of breast cancer. JAMA. 2008;299(18):2151-63.

35. • Chae EY, Cha JH, Shin HJ, Choi WJ, Kim HH. Reassessment and follow-up results of BI-RADS category 3 lesions detected on screening breast ultrasound. AJR. 2016;206(3):666-72. The frequency of ultrasound BI-RADS 3 was high (14.6\%) while the malignancy rate was very low $(0.7 \%)$, particularly for patients with normal mammograms. When the ACRIN 6666 protocol was strictly applied, nearly $20 \%$ of lesions were re-categorized. No malignancy was found in 213 reassessed BI-RADS category 2 lesions and 178 of these 213 lesions were downgraded because they were anechoic consistent with simple cysts. Multiplicity and analysis of lesion echogenicity and margin should be scrutinized to keep the rate of BI-RADS 3 low.

36. Chae EY, Kim HH, Cha JH, Shin HJ, Kim H. Evaluation of screening whole-breast sonography as a supplemental tool in conjunction with mammography in women with dense breasts. J Ultrasound Med. 2013;32(9):1573-8.

37. Barr RG, Zhang Z, Cormack JB, Mendelson EB, Berg WA. Probably benign lesions at screening breast US in a population 
with elevated risk: prevalence and rate of malignancy in the ACRIN 6666 trial. Radiology. 2013;269(3):701-12.

38. Hong AS, Rosen EL, Soo MS, Baker JA. BI-RADS for sonography: positive and negative predictive values of sonographic features. AJR. 2005;184(4):1260-5.

39. Raza S, Goldkamp AL, Chikarmane SA, Birdwell RL. US of breast masses categorized as BI-RADS 3, 4, and 5: pictorial review of factors influencing clinical management. Radiographics. 2010;30(5):1199-213.

40. Raza S, Chikarmane SA, Neilsen SS, Zorn LM, Birdwell RL. BIRADS 3, 4, and 5 lesions: value of US in management-followup and outcome. Radiology. 2008;248(3):773-81.

41. Marcon M, Frauenfelder T, Becker AS, Dedes KJ, Boss A. First ultrasound diagnosis of BI-RADS 3 lesions in young patients: can 6-months follow-up be sufficient to assess stability? Eur J Radiol. 2017;89:226-33.

42. Soo MS, Rosen EL, Baker JA, Vo TT, Boyd BA. Negative predictive value of sonography with mammography in patients with palpable breast lesions. AJR. 2001;177(5):1167-70.

43. Stavros AT, Thickman D, Rapp CL, Dennis MA, Parker SH, Sisney GA. Solid breast nodules: use of sonography to distinguish between benign and malignant lesions. Radiology. 1995;196(1):123-34.

44. Greenwood HI, Lee AY, Lobach IV, Carpentier BM, Freimanis RI, Strachowski LM. Clustered microcysts on breast ultrasound: what is an appropriate management recommendation? AJR. 2017;209:395-9.

45. Dennis MA, Parker SH, Klaus AJ, Stavros AT, Kaske TI, Clark SB. Breast biopsy avoidance: the value of normal mammograms and normal sonograms in the setting of a palpable lump. Radiology. 2001;219(1):186-91.

46. Berg WA, Zhang Z, Cormack JB, Mendelson EB. Multiple bilateral circumscribed masses at screening breast US: consider annual follow-up. Radiology. 2013;268(3):673-83.

47. Gordon PB, Gagnon FA, Lanzkowsky L. Solid breast masses diagnosed as fibroadenoma at fine-needle aspiration biopsy: acceptable rates of growth at long-term follow-up. Radiology. 2003;229(1):233-8.

48. Jang JY, Kim SM, Kim JH, Jang M, La Yun B, Lee JY, et al. Clinical significance of interval changes in breast lesions initially categorized as probably benign on breast ultrasound. Medicine. 2017;96(12):e6415.

49. Price ER, Sickles EA, Yitta S, Brasic N, Yeh MJ, Allen IE, et al. Use of the probably benign (BI-RADS category 3 ) assessment for masses on breast MRI: is it transferable to general clinical practice? Breast J. 2017. https://doi.org/10.1111/tbj.12865.

50. Mahoney MC, Gatsonis C, Hanna L, DeMartini WB, Lehman C. Positive predictive value of BI-RADS MR imaging. Radiology. 2012;264(1):51-8.

51. Liberman L, Morris EA, Dershaw DD, Abramson AF, Tan LK. MR imaging of the ipsilateral breast in women with percutaneously proven breast cancer. AJR. 2003;180(4):901-10.

52. Liberman L, Morris EA, Kim CM, Kaplan JB, Abramson AF, Menell JH, et al. MR imaging findings in the contralateral breast of women with recently diagnosed breast cancer. AJR. 2003;180(2):333-41.

53. • Houssami N, Ciatto S, Macaskill P, Lord SJ, Warren RM, Dixon JM, et al. Accuracy and surgical impact of magnetic resonance imaging in breast cancer staging: systematic review and meta-analysis in detection of multifocal and multicentric cancer. J Clin Oncol. 2008;26(19):3248-58. BI-RADS 3 is appropriate for a newly diagnosed mass with round or oval shape, circumscribed margins and type I or type II curve. BI-RADS 3 may be assigned to nonmass enhancement with focal or regional distribution and homogeneous internal enhancement. Any interval change in size, morphology or enhancement is regarded as indicative of malignancy.
54. Spick C, Szolar DH, Baltzer PA, Tillich M, Reittner P, Preidler $\mathrm{KW}$, et al. Rate of malignancy in MRI-detected probably benign (BI-RADS 3) lesions. AJR. 2014;202(3):684-9.

55. Yuen S, Uematsu T, Kasami M, Tanaka K, Kimura K, Sanuki J, et al. Breast carcinomas with strong high-signal intensity on T2weighted MR images: pathological characteristics and differential diagnosis. J Magn Reson Imaging. 2007;25(3):502-10.

56. Okafuji T, Yabuuchi H, Sakai S, Soeda H, Matsuo Y, Inoue T, et al. MR imaging features of pure mucinous carcinoma of the breast. Eur J Radiol. 2006;60(3):405-13.

57. Schnall MD, Blume J, Bluemke DA, DeAngelis GA, DeBruhl N, Harms S, et al. Diagnostic architectural and dynamic features at breast MR imaging: multicenter study. Radiology. 2006;238(1):42-53.

58. Bahrs SD, Baur A, Hattermann V, Hahn M, Vogel U, Claussen $\mathrm{CD}$, et al. BI-RADS ${ }^{\circledR} 3$ lesions at contrast-enhanced breast MRI: is an initial short-interval follow-up necessary? Acta Radiol. 2014;55(3):260-5.

59. Eby PR, DeMartini WB, Gutierrez RL, Saini MH, Peacock S, Lehman CD. Characteristics of probably benign breast MRI lesions. AJR. 2009;193(3):861-7.

60. Ha R, Sung J, Lee C, Comstock C, Wynn R, Morris E. Characteristics and outcome of enhancing foci followed on breast MRI with management implications. Clin Radiol. 2014;69(7):715-20.

61. Liberman L, Morris EA, Dershaw DD, Abramson AF, Tan LK. Ductal enhancement on MR imaging of the breast. AJR. 2003;181(2):519-25.

62. Sung JS, Lee CH, Morris EA, Comstock CE, Dershaw DD. Patient follow-up after concordant histologically benign imaging-guided biopsy of MRI-detected lesions. AJR. 2012;198(6):1464-9.

63. Li J, Dershaw DD, Lee CH, Kaplan J, Morris EA. MRI follow-up after concordant, histologically benign diagnosis of breast lesions sampled by MRI-guided biopsy. AJR. 2009;193(3):850-5.

64. Lee CH, Dershaw DD, Kopans D, Evans P, Monsees B, Monticciolo D, et al. Breast cancer screening with imaging: recommendations from the Society of Breast Imaging and the ACR on the use of mammography, breast MRI, breast ultrasound, and other technologies for the detection of clinically occult breast cancer. J Am Coll Radiol. 2010;7(1):18-27.

65. Benndorf $\mathrm{M}, \mathrm{Wu} \mathrm{Y}$, Burnside ES. A history of breast cancer and older age allow risk stratification of mammographic BI-RADS 3 ratings in the diagnostic setting. Clin Imaging. 2016;40(2):200-4.

66. Linda A, Zuiani C, Londero V, Di Gaetano E, Dal Col A, Girometti R, et al. Role of magnetic resonance imaging in probably benign (BI-RADS category 3 ) microcalcifications of the breast. Radiol Med. 2014;119(6):393-9.

67. Cho N, Lim J, Moon WK. Usefulness of ultrasound elastography in reducing the number of breast imaging reporting and data system category 3 lesions on ultrasonography. Ultrasonography. 2014;33(2):98-104.

68. Dijkstra H, Dorrius MD, Wielema M, Pijnappel RM, Oudkerk M, Sijens PE. Quantitative DWI implemented after DCE-MRI yields increased specificity for BI-RADS 3 and 4 breast lesions. J Magn Reson Imaging. 2016;44(6):1642-9.

69. Lee AY, Joe BN, Price ER. The predicament of the probably benign breast MRI: should we rely on intuition? Breast J. 2017;23(5):501-3.

70. Knogler T, Homolka P, Hoernig M, Leithner R, Langs G, Waitzbauer M, et al. Application of BI-RADS descriptors in contrast-enhanced dual-energy mammography: comparison with MRI. Breast Care. 2017;12(4):212-6.

71. Conners AL, Hruska CB, Tortorelli CL, Maxwell RW, Rhodes DJ, Boughey JC, et al. Lexicon for standardized interpretation of gamma camera molecular breast imaging: observer agreement and diagnostic accuracy. Eur $\mathrm{J}$ Nucl Med Mol Imaging. 2012;39(6):971-82. 
72. Conners AL, Maxwell RW, Tortorelli CL, Hruska CB, Rhodes DJ, Boughey JC, et al. Gamma camera breast imaging lexicon. AJR. 2012;199(6):W767-74.

73. Kuhl CK, Schmutzler RK, Leutner CC, Kempe A, Wardelmann E, Hocke A, et al. Breast MR imaging screening in 192 women proved or suspected to be carriers of a breast cancer susceptibility gene: preliminary results. Radiology. 2000;215(1):267-79.

74. Liberman L, Morris EA, Benton CL, Abramson AF, Dershaw DD. Probably benign lesions at breast magnetic resonance imaging: preliminary experience in high-risk women. Cancer. 2003;98(2):377-88.

75. Kriege M, Brekelmans CT, Boetes C, Besnard PE, Zonderland HM, Obdeijn IM, et al. Efficacy of MRI and mammography for breast-cancer screening in women with a familial or genetic predisposition. N Engl J Med. 2004;351(5):427-37.

76. Hartman AR, Daniel BL, Kurian AW, Mills MA, Nowels KW, Dirbas FM, et al. Breast magnetic resonance image screening and ductal lavage in women at high genetic risk for breast carcinoma. Cancer. 2004;100(3):479-89.

77. Sadowski EA, Kelcz F. Frequency of malignancy in lesions classified as probably benign after dynamic contrast-enhanced breast MRI examination. J Magn Reson Imaging. 2005;21(5):556-64.

78. Kuhl CK, Schrading S, Leutner CC, Morakkabati-Spitz N, Wardelmann E, Fimmers R, et al. Mammography, breast ultrasound, and magnetic resonance imaging for surveillance of women at high familial risk for breast cancer. J Clin Oncol. 2005;23(33):8469-76.
79. Eby PR, Demartini WB, Peacock S, Rosen EL, Lauro B, Lehman $\mathrm{CD}$. Cancer yield of probably benign breast MR examinations. J Magn Reson Imaging. 2007;26(4):950-5.

80. Weinstein SP, Hanna LG, Gatsonis C, Schnall MD, Rosen MA, Lehman CD. Frequency of malignancy seen in probably benign lesions at contrast-enhanced breast MR imaging: findings from ACRIN 6667. Radiology. 2010;255(3):731-7.

81. Hauth E, Umutlu L, Kümmel S, Kimmig R, Forsting M. Followup of probably benign lesions (BI-RADS 3 category) in breast MR imaging. Breast J. 2010;16(3):297-304.

82. Lourenco AP, Chung MT, Mainiero MB. Probably benign breast MRI lesions: frequency, lesion type, and rate of malignancy. J Magn Reson Imaging. 2014;39(4):789-94.

83. • Grimm LJ, Anderson AL, Baker JA, Johnson KS, Walsh R, Yoon SC, et al. Frequency of malignancy and imaging characteristics of probably benign lesions seen at Breast MRI. AJR. 2015;205(2):442-7. The cancer rate for BI-RADS 3 lesions was $2.5 \%$ and all cancers were in women with a genetic mutation or personal history of breast cancer.

84. Chikarmane SA, Birdwell RL, Poole PS, Sippo DA, Giess CS. Characteristics, malignancy rate, and follow-up of BI-RADS category 3 Lesions identified at breast MR imaging: implications for MR image interpretation and management. Radiology. 2016;280(3):707-15. 